\title{
Vaccination as a Control of Infectious Diseases
}

\section{Baafi J*, Darko 10 and Asenso FW}

Department of Mathematics and Science Education, Valley View University Techiman Campus, Ghana

\begin{abstract}
This project develops the SIR model of infectious diseases and uses it to study vaccination as a control strategy used to eradicate them. Vaccination combats the disease by offering immunity against future infection. Analytic expressions are obtained for key parameters such as the minimum vaccination level required. Numerical simulations are used to illustrate the main results.
\end{abstract}

Keywords: Epidemic models; Infectious diseases; Vaccination; Reproduction number; Stability

\section{Introduction}

Infectious diseases are caused by pathogenic micro-organisms, such as bacteria, viruses, parasites or fungi; the diseases can be spread, directly or indirectly, from one person to another (Health topics, 2015). Infectious diseases are among the leading cause of death in the human population worldwide. About 20 million people are affected by measles each year, primarily in the developing areas of Africa and Asia (Measles, 2015). During the 20th century, smallpox was responsible for an estimated $300\{500$ million deaths. As recently as 1967 , the World Health Organization (WHO) estimated that 15 million people contracted the disease and that two million died in that year (Smallpox, 2015). Polio at its peak in the 1940s and 1950s would paralyse or kill over half a million people worldwide every year (Poliomyelitis) [1]. Medicine has advanced over the years, but infectious disease outbreaks still pose a strong treat to humans. The most successful strategy in controlling infectious diseases has been vaccination (Vaccine, 2015).

Vaccination is the administration of antigenic material (a vaccine) to stimulate an individual's immune system to develop adaptive immunity to a pathogen. Vaccines can prevent morbidity from infection [2]. The effectiveness of vaccination has been widely studied and verified; for example, the influenza vaccine, the HPV (human papillomavirus) vaccine, and the chicken pox vaccine. Vaccination is the most effective method of preventing infectious diseases; widespread immunity due to vaccination is largely responsible for the worldwide eradication of smallpox (Edward Jenner in 1796) and the restriction of diseases such as polio, measles, and tetanus from much of the world [3-8]. The World Health Organization reports that licensed vaccines are currently available to prevent or contribute to the prevention and control of twenty-five infections (Vaccination, 2015). Vaccination operates by reducing the number of susceptible individuals in the population (Keeling and Rohani, 2008).

The question people normally ask in the midst of infection is this: Can the disease be eradicated or controlled? If the disease is always there, what proportion of the population will have it? Mathematical models attempt to provide answers to these questions. The SIR model in epidemiological modelling gives a simple dynamic description of three interacting populations, the susceptible, the infected, and the recovered. Though it is very simple, this model exhibits the basic structure associated to the spread of a disease in a population. There have been a lot of modifications to the basic SIR model to study more complex diseases and infection mechanisms as well as control measures (Sudipa et al. 2014). In this work, we discuss the equilibrium and stability analysis of a general susceptible-infected-recovered (SIR) epidemic model of infectious disease. We study both disease-free equilibrium and the endemic equilibrium of the afore-mentioned model $[9,10]$. We also discuss the basic reproduction number $\mathrm{R}_{0}$. We analyse vaccination as an intervention. We consider both the perfect and imperfect vaccines. This work also seeks to address the question, what proportion of the population should be vaccinated to control or eradicate the disease?

The paper is organized as follows: In Section 2, we present a formulation of general epidemic model and carry out the equilibrium and stability analysis of the model [11-15]. We also discuss the reproduction number and some numerical simulations. In Section 3, we carry out analysis for vaccination based on the model in Section 2. Section 4 contains the analysis of the imperfect vaccine and booster. Conclusion of this report is given in Section 5. The report is based mostly on the books (Keeling and Rohani, 2008) and (Britton, 2003).

\section{The SIR Model}

In this model, the total population $(\mathrm{N})$ is categorised into three compartments namely, the susceptible (S), the infective (I), and the recovered $(\mathrm{R})$. The susceptible are the uninfected individuals, but are able to become infected if exposed. The infective individuals are those with the disease and can transmit the infection to other susceptible individuals [16-18]. The recovered comprises of individuals who have been recovered from the infection and are immune to reinfection. At any given time ( $t$ ), the number of individuals in the susceptible, infective and recovery class is denoted by $\mathrm{S}(\mathrm{t}), \mathrm{I}(\mathrm{t})$ and $\mathrm{R}(\mathrm{t})$, respectively (Britton, 2003).

\section{Some assumptions underlying the model}

The model makes the following assumptions:

1. The population is fixed.

2. The birth rate is equal to the death rate and there is no disease related death.

${ }^{*}$ Corresponding author: Joseph Baafi, Department of Mathematics and Science Education, Valley View University Techiman Campus, Ghana, Tel: 0327010974 E-mail: jbaafi@aims.edu.gh

Received July 10, 2017; Accepted July 31, 2017; Published August 07, 2017

Citation: Baafi J, Darko IO, Asenso FW (2017) Vaccination as a Control of Infectious Diseases. J Appl Computat Math 6: 357. doi: 10.4172/2168-9679.1000357

Copyright: (c) 2017 Baafi J, et al. This is an open-access article distributed under the terms of the Creative Commons Attribution License, which permits unrestricted use, distribution, and reproduction in any medium, provided the original author and source are credited. 
3. Individuals leave the infective class either by recovery (and move to class R) or due to natural death.

4. Age, sex, status and race do not affect the probability of being infected.

5. All births enter the susceptible class.

6. Recovery confers immunity.

\section{The compartmental model}

SIR compartmental model indicating the movement of individuals from one class to the other.

Putting all these assumptions together with the corresponding notations, the above model as seen in Figure 1 can be represented mathematically by the following system of differential equations:

$\mathrm{S}=\mathrm{bn}-\beta \mathrm{si}-\mathrm{bs}$,

$\mathrm{I}=\beta \mathrm{SI}-\gamma \mathrm{I}-\mathrm{bI}$,

$\mathrm{R}=\gamma \mathrm{i}-\mathrm{bR}$,

With the non-negativity requirements of the initial conditions:

$\mathrm{S}(0) \geq 0, \mathrm{I}(0) \geq 0, \mathrm{R}(0) \geq 0$.

The parameter $b$ is the natural birth or death rate, is the effective contact rate between susceptible and infected individuals and is the recovery rate of an infected individual. $\mathrm{N}$ is the total population under consideration. Thus the total population density is given by $\mathrm{S}(\mathrm{t})+\mathrm{I}(\mathrm{t})+\mathrm{R}(\mathrm{t})=\mathrm{N}(\mathrm{t})$. Adding the three equations, we see that

$\mathrm{N}=\mathrm{S}+\mathrm{I}+\mathrm{R}=0$;

so that $\mathrm{N}(\mathrm{t})=\mathrm{N}(0)$ and the population size is constant.

As well as having positive initial data (2), the solution lies in the set

$\{(\mathrm{S}, \mathrm{I}, \mathrm{R}) ; \mathrm{S} \geq 0, \mathrm{I} \geq 0, \mathrm{R} \geq 0, \mathrm{~S}+\mathrm{I}+\mathrm{R}+\mathrm{N}\}$

It is easy to check that for all $t>0$,

$\mathrm{S}(\mathrm{t})>0, \mathrm{I}(\mathrm{t})>0, \mathrm{R}(\mathrm{t})>0$.

so that the solution remains in the above set.

\section{The basic reproduction number}

Now we determine the reproduction number $\mathrm{R}_{0}$. This is an important quantity which determines whether a disease persists or dies

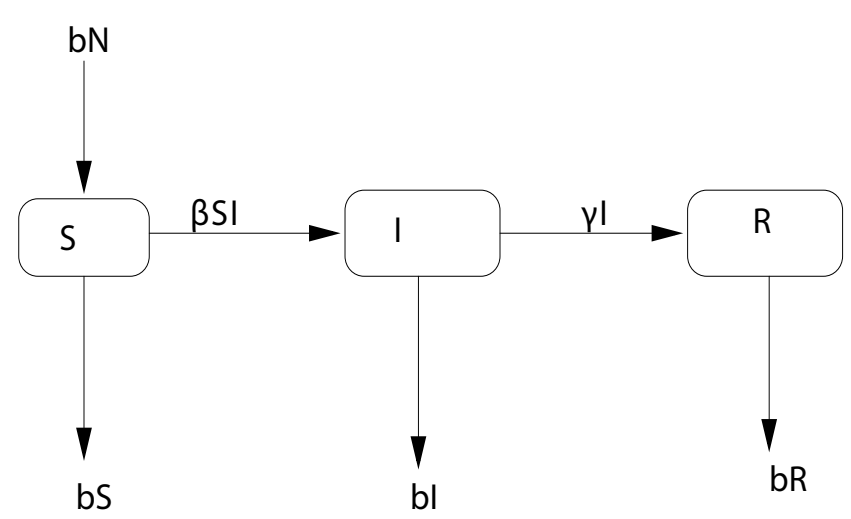

Figure 1: SIR compartmental model indicating the movement of individuals from one class to the other. out. It is defined as the average number of secondary cases arising from a single infective introduced into an entirely susceptible population of size N (Keeling and Rohani, 2008). The single infected individual makes $\beta \mathrm{N}$ contacts per unit time.

Also, the expected length of time that an infective remains in that state is.

Hence

$R_{0}=\frac{\beta N}{\gamma+\mathrm{b}}$

We show later that $\mathrm{R}_{0}$ is the critical parameter in determining the stability of equilibria for the above system.

\section{Equilibria}

We want to investigate the dynamics of the disease in the long run. We do this by considering what happens when the system is at equilibrium. At the equilibrium, we set all equations (14) in the system to zero and find the values of the variables $S^{*}, I^{*}, R^{*}$ which we now denote as S; I; R. We do this in the following result.

\section{Theorem 4.1}

The equilibrium points of the system $(14)$ are $(\mathrm{N}, 0,0)$ and

$$
\left(\frac{N}{R_{0}}, \frac{b}{\beta}\left(\mathrm{R}_{0}-1\right)-\frac{N}{R_{0}}-\frac{b}{\beta}\left(\mathrm{R}_{0}-1\right)\right)
$$

Proof.

We set all equations to zero

bN- $\beta S I-b S=0$,

$\beta S I-\gamma I-b I=0$,

$\gamma \mathrm{I}-\mathrm{Br}=0$

If $\mathrm{I}=0$, the second equation in (4) holds. Using $\mathrm{I}=0$ we obtain

$$
\mathrm{R}=0, \mathrm{bN}-\mathrm{Bs}=0
$$

Thus we have the equilibrium point

$$
\left(S_{1}^{*}, I_{1}^{*}, R_{1}^{*}\right)=(\mathrm{N}, 0,0)
$$

This is called the disease-free equilibrium point. At the disease free equilibrium, the disease no longer exists and the entire population is made up of all susceptible individuals as can be seen from the calculation.

If $\mathrm{I}=0$, then from the second equation in (4),

$\beta S=\gamma+b$

so that

$S=\frac{\gamma+b}{\beta}$

Using this value of S we have,

$b N-\frac{\beta N I}{R_{0}}-\frac{b N}{R_{0}} \Rightarrow I=\frac{b}{\beta}\left(\mathrm{R}_{0}-1\right)$

Now $\mathrm{R}$ is given by

$\mathrm{R}=\mathrm{N}-\frac{N}{R_{0}}-\frac{b}{\beta}\left(\mathrm{R}_{0}-1\right)$

For this to make biological sense we must have $R_{0}>1$. This is 
because population variables cannot be negative. Hence we have the other equilibrium point as:

$$
\left(\mathrm{S}_{2}^{*}, \mathrm{I}_{2}^{*}, R_{2}^{*}\right)=\left(\frac{N}{R_{0}}, \frac{b}{\beta}\left(\mathrm{R}_{0}-1\right), \mathrm{N}-\frac{N}{R_{0}}\left(\mathrm{R}_{0}-1\right)\right)
$$

This is called the endemic equilibrium point. If this equilibrium is stable, then the disease is not eradicated.

Let $\mathrm{A}$ be the mean time an individual remains susceptible. If $\mathrm{R}_{0}>1$, we use the above calculation to estimate A. Ignoring the small death rate, A will be equal to the inverse of the strength of infection, that is $(\beta \mathrm{I})^{-1}$. Using the equilibrium value for I in (7), we obtain an approximation to the mean age at infection:

$$
A \approx \frac{1}{b\left(\mathrm{R}_{0}-1\right)}
$$

The life expectancy $\mathrm{L}$ of the host is $\mathrm{L}=\mathrm{b}^{-1}$ so we have that

$$
R_{0}-1 \approx L A^{-1}
$$

For many animal and human infections, it is possible to measure $\mathrm{L}$ and $\mathrm{A}$ from data. We can then use the above expressions to estimate model parameters.

\section{Stability analysis}

Now we investigate the stability of these equilibrium points as follows:

\section{Theorem 4.2}

If $\mathrm{R}_{0}<1$, then the disease free equilibrium $(\mathrm{N}, 0,0)$ is stable.

Proof.

The Jacobian matrix of the system (14) is given by:

$$
J=\left(\begin{array}{ccc}
-\beta I^{*}-b & -\beta S^{*} & 0 \\
\beta I^{*} & -\beta S^{*}-(\gamma+\mathrm{b}) & 0 \\
0 & \gamma & -b
\end{array}\right)
$$

We evaluate this at the disease-free equilibrium.

The Jacobian matrix evaluated at the disease-free equilibrium gives:

$$
J(\mathrm{~N}, 0,0)=\left(\begin{array}{ccc}
-b & -\beta N & 0 \\
0 & -\beta N^{*}-(\gamma+\mathrm{b}) & 0 \\
0 & \gamma & -b
\end{array}\right)
$$

We compute the eigenvalues of this matrix as follows:

$$
\begin{aligned}
& \left|\begin{array}{ccc}
-b-\lambda & -\beta N & 0 \\
0 & -\beta N^{*}-(\gamma+\mathrm{b}) & 0 \\
0 & \gamma & -b-\lambda
\end{array}\right|=0 \\
& \Rightarrow(-b-\lambda)(\beta \mathrm{N}-(\gamma+b)-\lambda)(-\mathrm{b}-\lambda)=0
\end{aligned}
$$

Thus are the eigenvalues at the disease-free equilibrium

$$
\lambda 1=-b, \lambda 2=-b, \lambda 3=\beta N-(\gamma+b) \text {, }
$$

For the equilibrium to be stable, it is required that all eigenvalues are negative (or zero). It can be seen clearly that for all the eigenvalues to be negative, we must have $\beta \mathrm{N}<(\gamma+\mathrm{b})$ which means that the reproductive number $\mathrm{R}_{0}<1$. Thus for $\mathrm{R}_{0}<1$, the disease-free equilibrium is stable.

\section{Theorem 4.3}

If $\mathrm{R}_{0}>1$, then the endemic equilibrium (EE) is stable.

\section{Proof.}

The Jacobian matrix at the endemic equilibrium (EE) is given by:

$$
J(E, E)=\left(\begin{array}{ccc}
-b R_{0} & \frac{-\beta N}{R_{0}} & 0 \\
b\left(\mathrm{R}_{0}-1\right) & \frac{\beta N}{R_{0}}-(\gamma+\mathrm{b}) & 0 \\
0 & \gamma & -b
\end{array}\right)
$$

Now we compute the eigenvalues of the endemic equilibrium point as follows:

$$
\begin{gathered}
\left(\begin{array}{ccc}
-b R_{0}-\lambda & \frac{-\beta N}{R_{0}} & 0 \\
b\left(\mathrm{R}_{0}-1\right) & \left(\frac{\beta N}{R_{0}}-(\gamma+\mathrm{b})-\lambda\right) & 0 \\
0 & \gamma & -b-\lambda
\end{array}\right)=0 \\
\text { The eigenvalues of this matrix are }
\end{gathered}
$$

$\lambda_{1}=-\mathrm{b}$,

And after performing some algebra, we get

$$
\lambda_{2,3 .}=\frac{b R_{0}}{2} \pm \frac{\sqrt{\left(b R_{0}\right)^{2}-4 B\left(\mathrm{R}_{0}-1\right)(\mathrm{b}+\gamma)}}{2}
$$

To complete the proof, we show that the real parts of $\lambda_{2^{\prime}{ }_{3}}$ are negative.

$\mathrm{D}=\left(\mathrm{bR} \mathrm{R}_{0}\right)^{2}-4 \mathrm{~b}\left(\mathrm{R}_{0}-1\right)(\mathrm{b}+\gamma)$.

If $\mathrm{D}$ is negative then the eigenvalues $\lambda_{2,3}$ are complex with

$\operatorname{Re}\left(\lambda_{2,3}\right)=\frac{-b R_{0}}{2}<0$

If $\mathrm{D}>0$ then since $\mathrm{R}_{0}>1, \sqrt{\mathrm{D}}<\mathrm{b} \mathrm{R}_{0}$ and both $\lambda_{2}$ and $\lambda_{3}$ are negative.

For many problems, $\left(\mathrm{bR}_{0}\right)^{2}$ is very small so that the term under the square root sign is approximately

$$
-4 b\left(\mathrm{R}_{0}-1\right)(\mathrm{b}+\gamma)
$$

The duration $\mathrm{B}$ of infectivity is approximately, $\mathrm{B}=(\mathrm{b}+\gamma)^{-1}$, so using eqn. (11) we can approximate eqn. (11) by

$-4(\mathrm{AB})^{-1}$.

Where $\mathrm{A}$ is given by eqn. (8).

From this, the eigenvalues are

$$
\lambda_{2,3} \approx-\frac{b R_{0}}{2} \pm \frac{i}{\sqrt{A B}}
$$

Since the eigenvalues are complex, the equilibrium is approached via damped oscillations. The period $\mathrm{T}$ of these oscillations is approximated by:

$$
T \approx 2 \pi \sqrt{A B}
$$

By identifying the period we are able to determine when the disease will be most prevalent and when there will be a lull in the number of infected individuals. This could be useful in controlling an outbreak of a disease.

If the reproduction number $\mathrm{R}_{0}>1$ is close to 1 , then from eqn. (12), the real parts of the eigenvalues are negative and small while the imaginary part is very large. For this case, the approach to equilibrium is very slow and the period of oscillation is very large. 
This completes our analysis of the SIR model. The results will provide the basis for our future investigations into vaccination and quarantine control methods. We have identified the parameter $\mathrm{R}_{0}$ which determines if we have an endemic or a disease-free outcome.

\section{Numerical simulations}

In this section, we show some numerical simulations which illustrate some of the results in this chapter. The calculations are all for the SIR model given by eqn. (14). A crucial parameter which governs the behaviour of the system is the reproduction number $\mathrm{R}_{0}$ which is given in eqn. (3). All the graphs have time (measured in years) on the horizontal axis and the proportion of the population who are either susceptible, infective or recovered is on the vertical axis. All simulations are produced using python (odeint) (Figure 2).

$\mathrm{b}=1 / 70, \beta=520, \gamma=365 / 7$.

The initial data is

$\mathrm{S}(0)=0.1, \mathrm{I}(0)=2.5 \times 10-4, \mathrm{R}(0)=0.9-2.5 \times 10^{-4}$.

A calculation shows that $\mathrm{R} 0=10$. From section 2.5 , the endemic equilibrium is stable, but the amplitude of the fluctuations declines over time as the system equilibrates (a "damped oscillator"). From (13) the period $\mathrm{T}$ of oscillation is approximated as:

\section{$\mathrm{T}=0.774 \pi=2.43$}

The simulation shows that there are approximately four oscillations every ten years which matches the above value of $\mathrm{T}$. This result is shown in Figure 3.

\section{Vaccination against an SIR Epidemic}

For many infectious diseases there has been much focus on vaccinating new borns or young infants. Let $p$ denote the fraction of new borns who are successfully vaccinated. The assumption is that the vaccine provides long-lasting immunity to the infection which prevents both transmission and disease

The question we want to analyse is this: What proportion of the population do we have to vaccinate in order to eradicate the disease?

\section{The SIR model with vaccination}

When we put this into the SIR model we get the following:

$$
\begin{aligned}
& S^{\prime}=b(1-p) N-\beta S I-b S \\
& I^{\prime}=\beta S I-\gamma I-b I \\
& R^{\prime}=\gamma I-b R+b p N
\end{aligned}
$$

This modification can be dynamically explored using a simple change of variables:

$S=(1-p) S_{1}, I=(1-p) I_{1} R=(1-\mathrm{p}) \mathrm{R}_{1}+p N$

These substitutions give rise to a new set of differential equations as follows

$$
\begin{aligned}
& (1-p) S_{1}^{\prime}=b(1-\mathrm{p}) \mathrm{N}-\beta(1-p)^{2} S_{1} I_{1}-b(1-\mathrm{p}) \mathrm{S}_{1} \\
& (1-p) I_{1}^{\prime}=\beta(1-\mathrm{p})^{2} \mathrm{~S}_{1} \mathrm{I}_{1}-\gamma(1-p) I_{1}-b(1-\mathrm{p}) \mathrm{I}_{1} \\
& (1-p) R_{1}^{\prime}=\gamma(1-\mathrm{p}) \mathrm{I}_{1}-b(1-p) \mathrm{R}_{1}-b p N+b \mathrm{pN}
\end{aligned}
$$

Dividing by 1-p gives:

$$
\begin{aligned}
& S_{1}^{\prime}=b N-(1-p) \beta S_{1} I_{1}-b S_{1} \\
& I_{1}^{\prime}=(1-p) \beta S_{1} I_{1}-\gamma I_{1}-b I_{1} \\
& R_{1}^{\prime}=\gamma I_{1}-b R_{1}
\end{aligned}
$$

It can be seen that this equation is same as the SIR model with $\beta$ replaced by $(1-\mathrm{p}) \beta$. Hence, we get a new $\mathrm{R}_{0}$ denoted by $\mathrm{R}_{0}^{*}$ by:

$$
R_{0}^{*}=\frac{(1-p) \beta N}{\gamma+b}=(1-p) R_{0}
$$

From the stability analysis carried out in Section 2.5, the diseasefree equilibrium is stable if $R_{0}^{*}<1$

Hence to prevent the disease from spreading, we need to ensure that

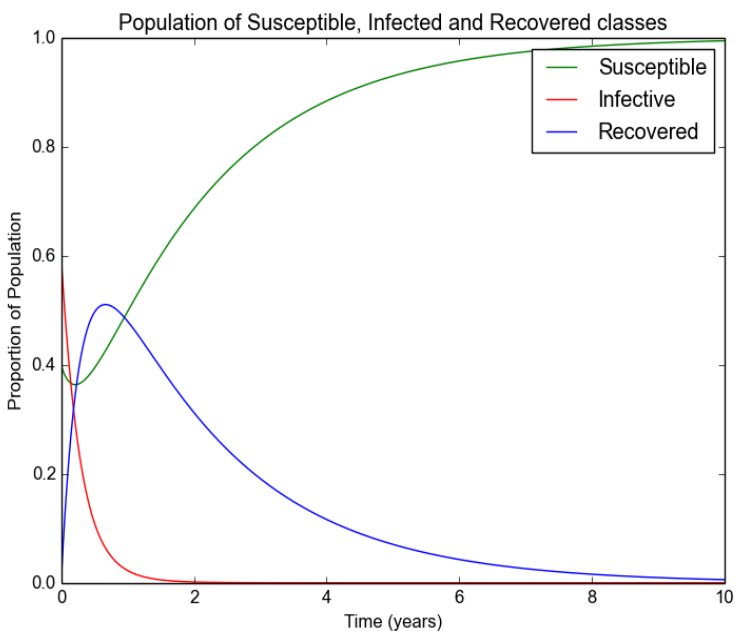

(a)

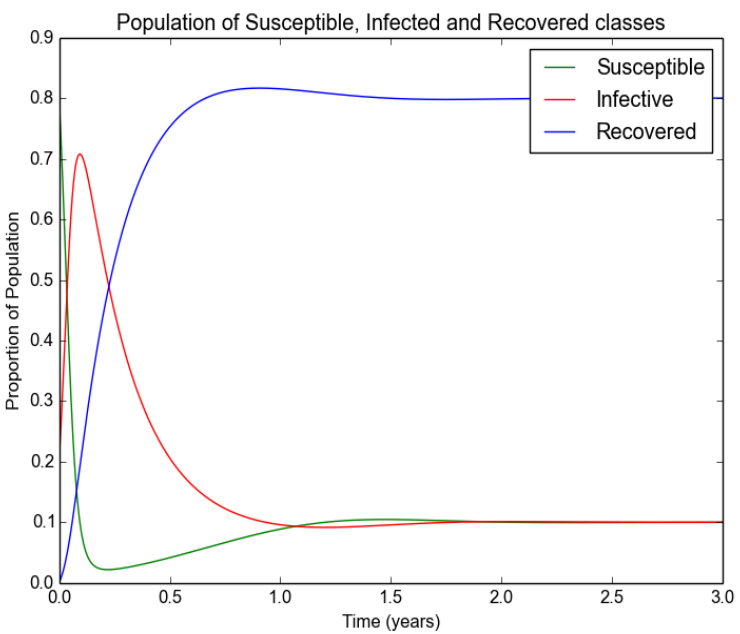

(b)

Figure 2: Numerical solutions of the SIR model (14) with $b=0.5, \beta=3, y=4, N=1, S(0)=0.4, I(0)=0.6, R(0)=0.0$ and $R 0=0.74<1$. By Theorem 2.2 the disease-free equilibrium is stable (a). Also, $b=0.5, \beta=45, y=4, N=1, s(0)=0.8, I(0)=0.2, R(0)=0$, and $R 0=10>1$. By Theorem 2.3 , the endemic equilibrium is stable (b). 


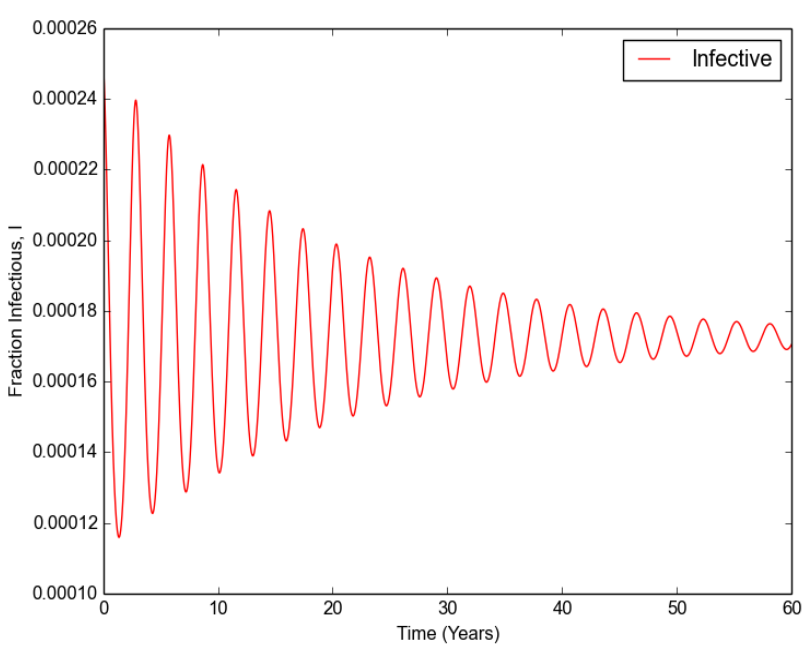

Figure 3: Dynamics for Example 2.1

\begin{tabular}{|l|l|l|}
\hline Infectious disease & $\boldsymbol{R}_{\mathbf{0}}$ & $\hat{P} \%$ \\
\hline Smallpox & $3-5$ & $67-80$ \\
\hline Measles & $12-13$ & 92 \\
\hline Whooping cough & $13-17$ & $92-94$ \\
\hline Chickenpox & $9-10$ & $89-90$ \\
\hline Mumps & $4-7$ & $75-86$ \\
\hline Diphtheria & $4-6$ & $75-83$ \\
\hline Poliomyelitis & 6 & 83 \\
\hline
\end{tabular}

Table 1: Table showing the relationship between $\boldsymbol{R}_{0}$ and $p$ for some disease.

$$
R_{0}^{*}=(1-p) R_{0}<1
$$

This is ensured by vaccinating a critical proportion $\hat{p}$ of the susceptible individuals (newborns).

$$
\text { We calculate } \hat{p} \text { by solving }
$$$$
R_{0}^{*}=(1-\hat{p}) R_{0}=1
$$

to get

$$
\hat{p}=1-\frac{1}{R_{0}}
$$

For the disease to be eradicated totally from the population, we need to vaccinate at least a proportion of the susceptibles $p$ with

$$
\hat{p}=1-\frac{1}{R_{0}}
$$

In order to eradicate an infection, not all individuals need to be vaccinated, as long as a critical proportion (determined by the reproduction number of the infection) have been afforded protection. This phenomenon is referred to as "herd immunity" (Keeling and Rohani, 2008).

Table 1 (taken from Britton, 2003) shows some infectious diseases with their estimated values of $R_{0}$ and ^ $p$. Most of these data are from England and Wales, USA or other developed countries. It is estimated that values of $\mathrm{R}_{0}$ in developing countries tend to be higher, especially in densely populated countries such as the Indian subcontinent. The values of R0 for smallpox given in Table 1 are for developing countries and they are surprisingly low. See Figure 4 for the relationship between $\mathrm{R} 0$ and the critical vaccination proportion Table 1.
Considering Poliomyelitis, we can see that for the disease to be eradicated, we need to successfully vaccinate at least $83 \%$ of the susceptible individuals especially new born babies. For the case of whooping cough, eradication of the disease from the population requires a successful vaccination of $94 \%$ of all susceptible individuals. This could be practically difficult especially in countries where finances play a major role in the number of people who receive the vaccines. We therefore need to explore other means of disease control which may be relatively cheaper. One such method is pulse vaccination (Keeling and Rohani, 2008) in which we repeatedly vaccinate the susceptible individuals $\mathrm{S}$ over a defined age range until the disease dies out. We will discuss this in the next section.

\section{Numerical Simulations of the SIR model with vaccination}

In this section, we show some numerical simulations which illustrate some of the results in this chapter. The calculations are all for the modified SIR with vaccination model given by eqn. (14). A crucial parameter $R_{0}^{*}$ given by eqn. (16) governs the behaviour of the system. Eradication of infection is ensured by vaccinating a threshold proportion of the susceptible individuals given in eqn. (17).

Example 5.1. In this example, all parameters are same as in Figure 2 in the previous section. To prevent the disease from spreading using eqn. (18), we vaccinate at least a critical proportion

\section{$\hat{p} 0.9$,}

of the susceptible individuals. The vaccine reduces the reproduction number $\mathrm{R}_{0}$ from $\mathrm{R}_{0}=10$ to $\mathrm{R}^{*}=0.9$. By Theorem 2.2 the disease-free equilibrium is stable. That is, the vaccine is eradicating the disease from the population under consideration. This result with $\mathrm{p}=\hat{p}$ is shown in Figure 5.

Example 5.2 This example again uses the same parameter values as 2.6 Figure 2. We consider the case where the proportion of susceptible individuals vaccinated

$$
\mathrm{p}=0.35<0.9=\hat{p},
$$

is less than the critical proportion $\hat{p}$ needed to eradicate the disease. The reproduction number $\mathrm{R}_{0}=10$ is reduced to

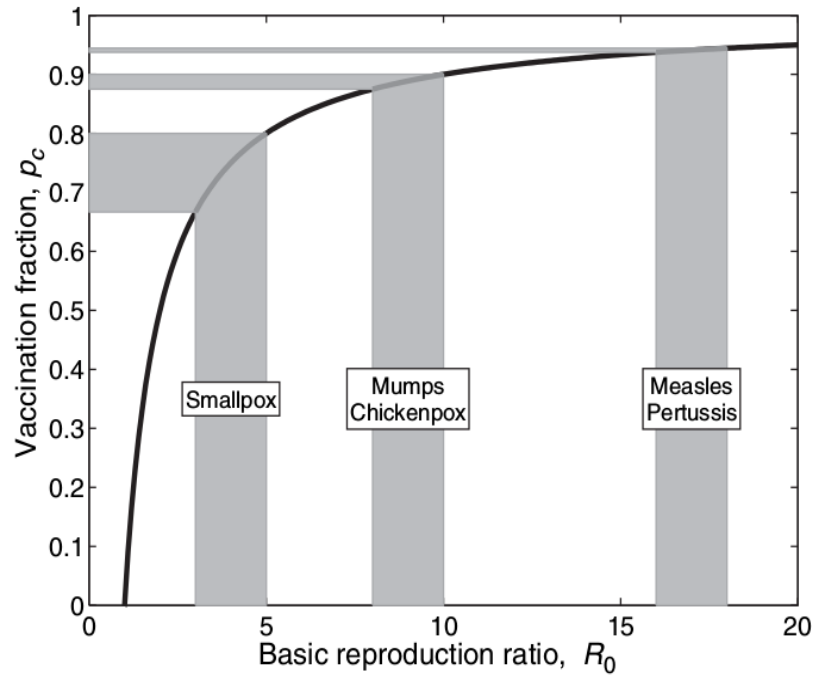

Figure 4: This figure shows the relationship between R0 and the fraction of susceptibles that needs to be vaccinated in order to eradicate the disease. This Figure is taken from (Keeling and Rohani, 2008). 
$\mathrm{R}^{*}=6.5>1$. By Theorem 2.3, the endemic equilibrium is stable, that is, the infection do not die out but the rate at which the infection spreads is reduced. In the long run, about $5 \%$ of the population is infected. Figure 6 illustrates this result.

We see that by implementing the vaccination program, the level of infected individual's decreases. It converges to an endemic equilibrium but at a lower level than Figure 2 (which has $\mathrm{p}=0$ ). This example shows how mathematical modelling play an important role in estimating how much a vaccination program can help. Public health authorities can use this information to see how the benefits match the cost of a vaccination program (Figure 6).

\section{Imperfect Vaccines and Boosting}

In the previous chapter, we showed that vaccines can dramatically reduce the number of new infectious cases. A major assumption was that the vaccines that were administered were $100 \%$ effective in

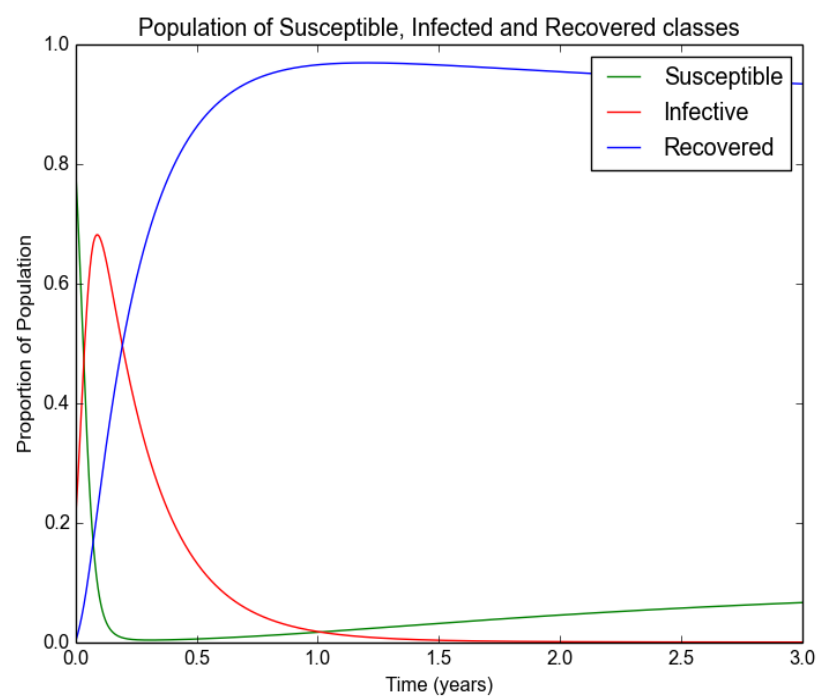

Figure 5: Dynamics for Example 3.1

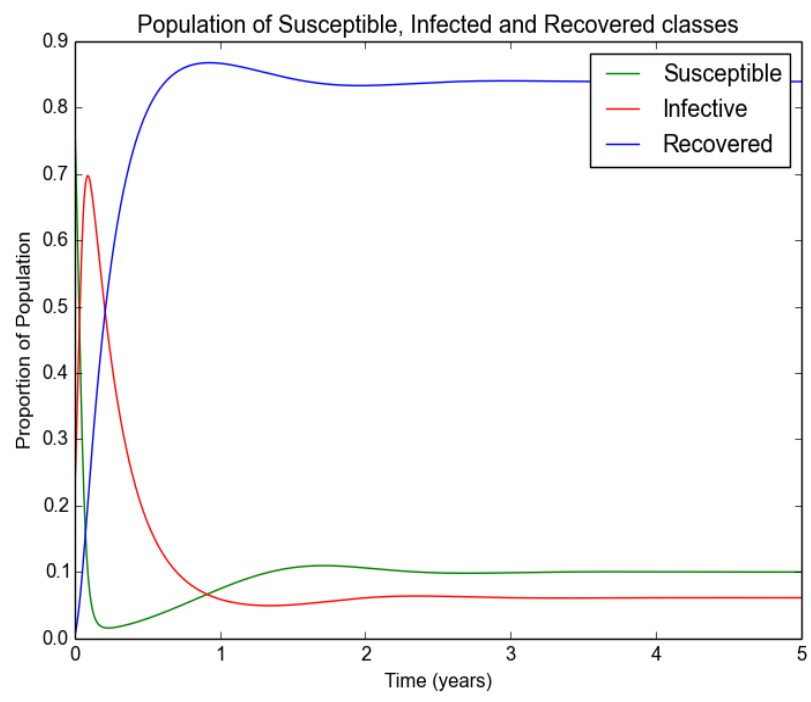

Figure 6: Dynamics for Example 3.2 providing permanent protection. However, the immunity obtained from a vaccine may not be complete and may wane with time. To prevent the resurgence of a disease, it can be advantageous to boost the initial vaccination (Keeling and Rohani, 2008).

\section{The model}

The model accounts for two aspects of an imperfect vaccine:

(i) Incomplete protection.

(ii) The decrease in vaccination-induced protection.

We modify the equations in Section 3 to take account of these aspects. We add a new class to the model in Section 3 so there are now four classes; $\mathrm{S}, \mathrm{S}_{\mathrm{v}}, \mathrm{I}$ and V. Here $\mathrm{S}$ is the susceptibles, $\mathrm{S}_{\mathrm{v}}$ is vaccinated, $\mathrm{I}$ is infectious. The class $\mathrm{V}$ is the booster vaccinated (or recovered) individuals who are immune for life. Figure 7 represents the dynamics in a flow diagram.

The parameter $a$ represents the amount that the initial vaccination failed to confer full immunity against the disease. The rate at which the immunity granted by the first vaccination decreases is the parameter $\delta$. The parameters $\theta$ and represent the rates of administration of the booster vaccine to the previously vaccinated and susceptible classes respectively. Other parameters are defined as in the previous chapters.

The following system of ODE's describe the model:

$$
\begin{aligned}
& S^{\prime \prime}=b(1-\mathrm{p}) \mathrm{N}-\beta \mathrm{SI}-\mathrm{bS}-\mathrm{cS}+\delta S_{v} \\
& S_{v}^{\prime}=b p \mathrm{~N}+c S-(1-\alpha) \beta \mathrm{S}_{w} \mathrm{I}-(\delta+\mathrm{b}+0) S_{v} \\
& I^{\prime}=\beta S I+(1-\alpha) \beta S_{v} I-(\mathrm{b}+\gamma) \mathrm{I} \\
& \mathrm{V}^{\prime}=\theta S_{v}+\gamma I-b V
\end{aligned}
$$

A calculation shows that:

$$
S^{\prime}+S_{v}^{\prime \prime}+I^{\prime}+V^{\prime}=0
$$

so the total population is constant and

$$
S+S+I+V=N
$$

We then have

$$
V=N-S-S_{v}-I
$$

Hence, we can reduce and analyse the first three equations in eqn. (19).

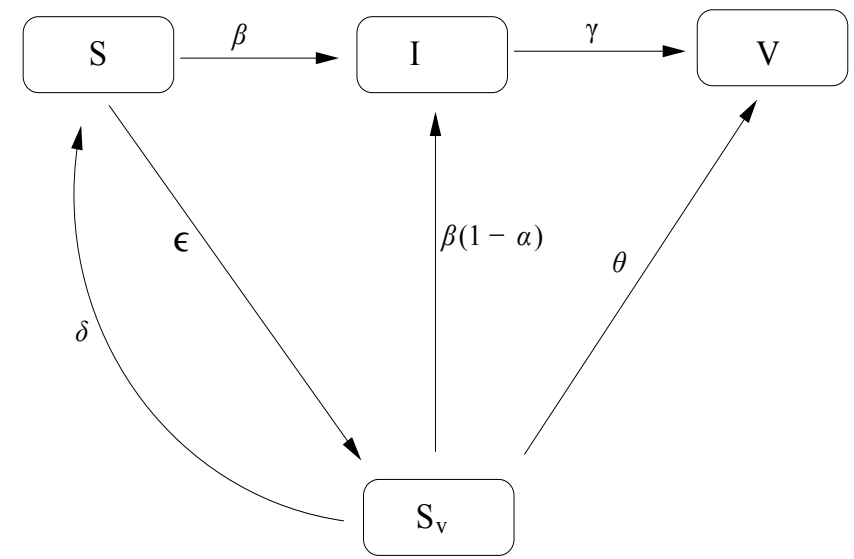

Figure 7: Simple SSvIV compartmental model which represents the dynamics in a flow diagram. 


\section{Disease eradication}

As in earlier chapters, the system has both a disease-free equilibrium and an endemic equilibrium. Analysis of these equilibrium solutions are more complicated than the previous model. Since we are interested in preventing an endemic spread of disease, we only study the stability of the disease-free equilibrium. To do this we need only to study the three dimensional system given by the first three equations in eqn. (19).

We find the disease-free equilibrium with $\mathrm{I}=0$. For this equilibrium: $\mathrm{b}(1-\mathrm{p}) \mathrm{N}-\mathrm{bS}-\in \mathrm{S}+\delta \mathrm{Sv}=0$,

$\mathrm{pbN}+\in \mathrm{S}-(\delta+\mathrm{b}+\theta) \mathrm{S}_{\mathrm{v}}=0$.

Solving these equations we obtain:

$$
\begin{aligned}
& S=\frac{b N[(\mathrm{~b}+\theta)(1-\mathrm{p})+\delta]}{(\mathrm{b}+\mathrm{c})(\mathrm{b}+\theta)+\delta \mathrm{b}} \\
& S_{v}=\frac{b N(\mathrm{bp}+\epsilon)}{(\mathrm{b}+\epsilon)(\mathrm{b}+\theta) \delta \mathrm{b}}
\end{aligned}
$$

We now study the stability of the disease-free equilibrium (DFE).

The Jacobian matrix at this equilibrium is given by:

$$
J(\mathrm{DFE})=\left(\begin{array}{ccc}
-b-c & \delta & -\beta S \\
c & -(\delta+\mathrm{b}+\theta) & -(1-\alpha) \beta \mathrm{S}_{v} \\
0 & 0 & \beta S+(1-\alpha) \beta \mathrm{S}_{v}-(\mathrm{b}+\gamma)
\end{array}\right)
$$

The eigenvalues of J(DFE) are as follows:

$\lambda 1=\beta S+(1-\alpha) \beta S_{v}-(b+\gamma)$,

And the solution to the quadratic

$$
\lambda^{2}+(2 b+\delta+\theta) \lambda+b(\delta+b+\theta+)+\in \theta=0 .
$$

Since the coefficients of the quadratic in eqn. (23) are positive, the solutions of the quadratic have negative real parts. It follows that the disease-free equilibrium will be stable if $\lambda_{1}<0$ and unstable if $\lambda_{1}>0$.

Using the expression for $\mathrm{S}$ and $\mathrm{Sv}$ given by eqn. (21), we can calculate the reproduction number $r_{0}$ so that we have stability if $r_{0}<1$ and instability if $r_{0}>1$. After some algebra we obtain:

$$
r_{0}=\frac{b \beta N[(\mathrm{~b}+\theta)(1-\mathrm{p})+\delta+(1-\alpha)(\mathrm{bp}+\epsilon)]}{[(\mathrm{b}+\epsilon)(\mathrm{b}+\theta)+\delta \mathrm{b}](\mathrm{b}+\gamma)}
$$

\section{Analysis of the reproduction number}

To eradicate the disease we require that $r_{0}<1$. In principle, this may be achieved by increasing the vaccination level $p$. However, this is not always feasible, so it is important to understand how $r_{0}$ depends on the other parameters.

To continue this analysis, it is useful to relate $\mathrm{r} 0$ in eqn. (24) to the reproduction number for a population that is completely susceptible, that is, no vaccination. This was the case considered in Section 2.3 for which

$$
R_{0}=\frac{\beta N}{b+\gamma}
$$

After some algebra, eqn. (24) can be rewritten as:

$$
r_{0}=\left(1-\frac{(\mathrm{bp}+\epsilon)(\mathrm{b} \alpha+\theta)}{(\mathrm{b}+\theta)(\mathrm{b}+\epsilon)+\mathrm{b} \delta}\right) R_{0}
$$

To understand the critical vaccination level that is needed to prevent the disease spreading, we consider the case when there is no boosters so that $\in=\theta=0$. This is the same as the vaccination model in Section 3 but with partial protection and waning immunity. Solving $\mathrm{r}_{0}=1$ for this case gives the critical vaccination level:

$$
p_{c}=\left(1-\frac{1}{R_{0}}\right)\left(\frac{b+\delta}{b \alpha}\right)
$$

so that $\mathrm{r}_{0}<1$ if $\mathrm{p}>\mathrm{p}_{\mathrm{c}}$. As a check, for a perfect vaccine we have that $\alpha=1$ and $\delta=0$ and $p c$ is equal to the value in eqn. (17).

We now use this result to show that it may be impossible to eradicate the disease. Suppose that the primary vaccine provides perfect immunity $(\alpha=1)$ and that this protection wanes with time $(\delta>$ $0)$. For this case

$$
p_{c}=\left(1-\frac{1}{R_{0}}\right)\left(\frac{b+\delta}{b}\right)
$$

For pc to be less than 1 we require

$$
\frac{b}{b+\delta}>1-\frac{1}{R_{0}}
$$

The LHS of the inequality in eqn. (28) is the fraction of the vaccinated individuals life during which they are protected from infection.

If, for example $\mathrm{R}_{0}=2$ then eradication is possible only when the vaccine protects individuals for more than half their life. Hence the need for booster vaccination.

\section{Numerical simulations of the SIR model with vaccines and boosting}

In this example, we assume the following parameter values:

$\mathrm{b}=0.5, \beta=19, \gamma=4=1, \alpha=0.90, \theta=1, \delta=0.05, \mathrm{~N}=1$,

with $\mathrm{p}$ to be chosen.

A calculation shows that if we have no vaccination the SIR model gives

$$
R_{0}=\frac{\beta N}{b+\gamma}=4.2
$$

so the endemic equilibrium is stable.

We now use equation (26) to calculate $r_{0}$ :

$$
r_{0}=\left(1-\frac{(0.5 p+1)(0.5 \times 0.90+1)}{(0.5+1)(0.5+1)+0.5 \times 0.05}\right) x 4.2
$$

To find the critical vaccination level we solve $r_{0}=1$ to get

$\mathrm{p}_{\mathrm{c}}=0.4$.

Taking $\mathrm{p}=0.5$ greater than $\mathrm{pc}$, with the above values of the other parameters we calculate that the reproduction number $R_{0}=4.2$ is reduced to $r_{0}=0.86$. Since $r_{0}$ is less than 1 , from Section 4.2 the disease free equilibrium is stable.

We numerically solve equation (19) with the above parameter values and $\mathrm{p}=0.5$. The result is shown in Figure 8 .

\section{Conclusion}

One motivation for studying mathematical models of infectious diseases is to improve methods for controlling and eradicating disease. The key equations for doing this, the SIR model was studied in Section 2 . The reproduction number $\mathrm{R}_{0}$ was derived and it was shown that if $\mathrm{R}_{0}<1$ then the disease will die out while if $\mathrm{R}_{0}>1$, the disease will spread. 


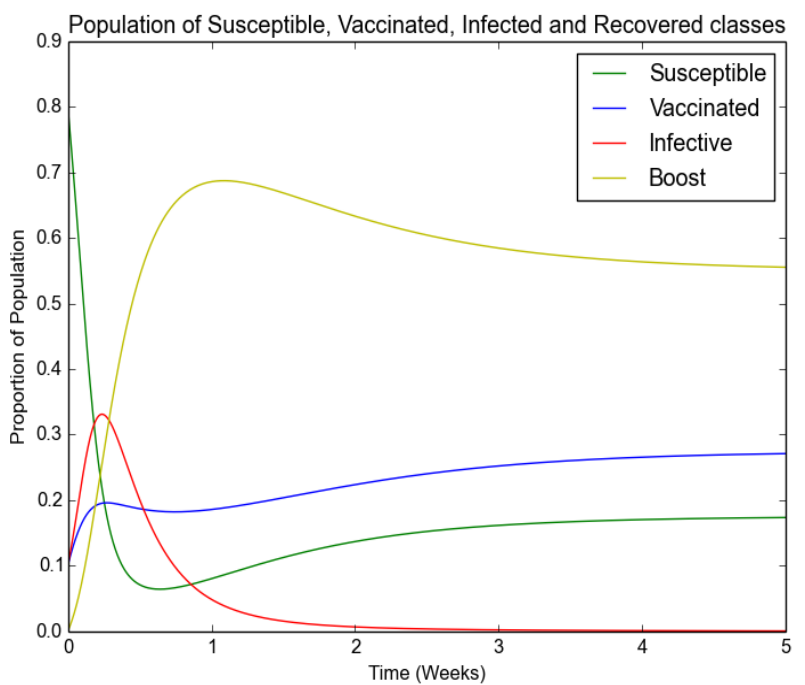

Figure 8: Dynamics in the imperfect vaccine with boost.

A list of the estimated values of $R_{0}$ was given in Table 1 . The aim of any control policy is to reduce $\mathrm{R}_{0}$ and to make it less than 1 if possible.

There are a number of limitations to the SIR model.

- Disease may spread spatially. To model this we would replace the ordinary differential equations in the SIR model by partial differential equations.

- Seasonality can be important in the study of some diseases. To model this we would replace some of the constant parameters in the SIR model by periodic functions of time.

- Some elements of the disease may be random and we would need to replace the deterministic SIR model by a stochastic differential equation.

In later sections we studied a method used to combat disease. In Section 3 we studied vaccination and we determined the minimum vaccination level needed to eradicate a disease. Not all vaccines are perfect, any may over incomplete protection. This was studied in Section 4. It was found that vaccination is an effective strategy to control infectious disease.

\section{References}

1. Keeling KJ and Pejman R (2008) Modeling infectious diseases in humans and animals.

2. Infectious diseases (2015) Health topics.

3. Measles (2015) Wikipedia, the Free Encyclopedia..

4. Smallpox (2015). Wikipedia, the Free Encyclopedia.

5. Poliomyelitis (2015) Wikipedia, the Free Encyclopedia.

6. Vaccine (2015) Wikipedia, the Free Encyclopedia.

7. Vaccination (2015) Wikipedia, the Free Encyclopedia.

8. Hethcote and Herbert W (2000) The mathematics of infectious diseases. SIAM review 42: 599-653.

9. Mathematical model (2015) Wikipedia, the Free Encyclopedia.

10. Chauhan S, Misra OP and Dhar J (2014) Stability analysis of SIR model with vaccination. American Journal of Computational and Applied Mathematics 4 17-23.

11. Nicholas FB (2003) Essential mathematical biology. Springer Science \& Business Media.

12. Murray JD (2002) Mathematical biology I: An Introduction, Interdisciplinary Applied Mathematics Mathematical Biology.

13. Tessa and Moussa $O$ (2006) Mathematical model for control of measles by vaccination. In Proceedings of Mali Symposium on Applied Sciences.

14. Meihong Q, Huan Q and Tian T (2010) Steady State Solution and Stability of an Age-Structured MSIQR Epidemic Model. Intelligent Information Management 2: 316-324.

15. Alexander ME, Moghadas SM, Rohani P and Summers AR (2005) Modelling the effect of a booster vaccination on disease epidemiology. Journal of mathematical biology 52: 290-306.

16. Ullah R, Zaman G and Islam S (2013) Stability analysis of a general SIR epidemic model. VFAST Transactions on Mathematics 1;16-20.

17. Brauer F (2008) Compartmental Models in Epidemiology. In Mathematical Epidemiology Springer 19-79.

18. Atkins TA (2010) Using Modeling and Simulation to Evaluate Disease Contro Measures. PhD thesis, University of Central Florida Orlando. 